\title{
Scattering of flexural waves in Euler-Bernoulli beams by short-range potentials Paper
}

Christiansen, Peter Leth; lermakova, Sofia V.; Gaididei, Yuri B.; Sørensen, Mads Peter

Published in:

Journal of Physics A: Mathematical and Theoretical

Link to article, DOI:

10.1088/1751-8121/aaa810

Publication date:

2018

Document Version

Peer reviewed version

Link back to DTU Orbit

Citation (APA):

Christiansen, P. L., lermakova, S. V., Gaididei, Y. B., \& Sørensen, M. P. (2018). Scattering of flexural waves in Euler-Bernoulli beams by short-range potentials: Paper. Journal of Physics A: Mathematical and Theoretical, 51(9), [095202]. https://doi.org/10.1088/1751-8121/aaa810

\section{General rights}

Copyright and moral rights for the publications made accessible in the public portal are retained by the authors and/or other copyright owners and it is a condition of accessing publications that users recognise and abide by the legal requirements associated with these rights.

- Users may download and print one copy of any publication from the public portal for the purpose of private study or research.

- You may not further distribute the material or use it for any profit-making activity or commercial gain

- You may freely distribute the URL identifying the publication in the public portal 


\title{
Scattering of flexural waves in Euler-Bernoulli beams by short-range potentials
}

\section{Peter L. Christiansen}

Department of Physics and Department of Applied Mathematics and Computer Science, Technical University of Denmark, DK-2800 Kongens Lyngby, Denmark

\section{Sofia V. Iermakova}

Bogolyubov Institute for Theoretical Physics, 03143 Kyiv, Ukraine

\section{Yuri B. Gaididei}

Bogolyubov Institute for Theoretical Physics, 03143 Kyiv, Ukraine

\section{Mads Peter Sørensen}

Department of Applied Mathematics and Computer Science, Technical University of Denmark, DK-2800 Kongens Lyngby, Denmark

\begin{abstract}
Time-harmonic flexural waves on a beam and on two elastically coupled beams with short-range localized imperfections in the mass distribution and in the position dependant coupling are considered. Thus scattering of an incident wave solution to the Euler-Bernoulli equation by a Dirac delta function and its derivative up to order three is studied, and the possible physical interpretations are outlined. Reflected, transmitted and evanescent waves exist, and their scattering data are determined. For $\delta(x)$ and $\delta^{\prime}(x)$, the scattering problem is solved by standard integration. For $\delta^{\prime \prime}(x)$ and $\delta^{\prime \prime \prime}(x)$, the standard integration procedure does not work and solutions are obtained by regularization. In the latter case the scatterer is in general nontransparent and only partially penetrable at discrete resonances. The first few of these as well as their scattering data are determined numerically.
\end{abstract}

PACS numbers: 46.70.He, 3.65.Nk, 11.10.Gh, 73.22.Dj 


\section{Introduction}

Models described by Schrödinger operators with singular zero-range potentials are widely discussed in the physical and mathematical literature starting with the pioneering work by Berezin and Faddeev [1]. For discussions see [2]- [5] and references therein. Analytical solutions using different regularizations of the singular potentials are obtained in [6]- [11].

Most of the aforementioned papers are devoted to studying the peculiar features of scattering of quantum particles by a short-range dipole-like potential $V_{1}(x)=\delta^{\prime}(x)$ in the framework of the Schrödinger equation

$$
-\frac{d^{2}}{d x^{2}} \psi+\sigma^{2} V_{1}(x) \psi=E \psi
$$

Here the prime stands for the derivative with respect to the spatial coordinate $x, E$ is the energy, $\sigma$ is a strength of the potential and $\delta^{\prime}(x)$ is the derivative of Dirac's delta function. By using a regularized version of the $\delta^{\prime}$-potential in the form

$$
V_{1}(x)=\frac{1}{\epsilon^{2}} \mathcal{V}_{1}\left(\frac{x}{\epsilon}\right)
$$

with $\mathcal{V}_{1}(\xi)$ being a smooth compactly supported function which satisfies the conditions

$$
\int_{-\infty}^{\infty} \mathcal{V}_{1}(\xi) d \xi=0, \quad \int_{-\infty}^{\infty} \xi \mathcal{V}_{1}(\xi) d \xi=-1
$$

Seba in Ref. [12] proved that in the limit $\epsilon \rightarrow 0$ the potential $V_{1}(x)$ (i.e. $\delta^{\prime}$-barrier) is totally nontransparent. By using a piece-wise constant compactly supported function $\mathcal{V}_{1}(\xi)$, it was shown in [9] that a $\delta^{\prime}$-resonant tunneling takes place: at some values of the potential strength $\sigma=\sigma_{n}(n=1,2, \ldots)$ the transmission across the dipole-like barrier is finite whereas for $\sigma \neq \sigma_{n}$ the barrier is completely opaque. The existence of nonzero transmission for a wide class of $\delta^{\prime}$-like regularizing sequences with compact support was confirmed in Refs. [13] - [15]. Several types of heterostructures consisting of few piece-wise constant $\delta$-approximating functions was considered quite recently in [16]. The two-scale squeezing of the heterostructure to one point as both the width of $\delta$ approximating functions and the distance between these functions simultaneously tend to zero was studied and a family of limit one-point interactions with resonant-tunnelling behaviour has been realized.

Eq. (1) with the potential $V_{1}(x)$ given by Eqs. (2) and (3) belongs to a family of equations

$$
(-1)^{n} \frac{d^{2 n}}{d x^{2 n}} \psi+\sigma^{2 n} V_{2 n-1}(x) \psi=E \psi, \quad n=1,2, \ldots,
$$

where $V_{2 n-1}(x)=\delta^{(2 n-1)}(x)$, and $\delta^{(n)}(x)$ is the $n$-th derivative of Dirac's delta function. Using the regularization

$$
V_{2 n-1}(x)=\frac{1}{\epsilon^{2 n}} \mathcal{V}_{2 n-1}\left(\frac{x}{\epsilon}\right) \rightarrow \delta^{(2 n-1)}(x), \quad \text { as } \quad \epsilon \rightarrow 0,
$$




$$
\int_{-\infty}^{\infty} \mathcal{V}_{n}(\xi) d \xi=0, \quad \int_{-\infty}^{\infty} \xi^{n} \mathcal{V}_{n}(\xi) d \xi=(-1)^{n} n !, \quad n=1,2, \ldots
$$

we see that all members of the family are characterized by the same singular behavior of the kinetic part (the first term on the l.h.s.) and the potential part (the second term on the l.h.s.) of the equations: by rescaling the spatial variable $\xi=x / \epsilon$, we get

$$
(-1)^{n} \frac{d^{2 n}}{d \xi^{2 n}} \psi+\sigma^{2 n} \mathcal{V}_{2 n-1}(\xi) \psi=\epsilon^{2 n} E \psi, \quad n=1,2, \ldots
$$

It is of interest therefore to clarify how the wave scattering modifies in the case of $n$-order differential equation with $n$-pole short-range potential. In this paper we consider the fourth-order differential equation with a $\delta^{\prime \prime \prime}(x)$ potential, $i$. e e. the case $n=2$. Such kind of problem arises in the mechanics of beams [17] which contain a short-range quadrupole defect. Note that the fourth order Euler-Bernoulli equation describing the deflection of beam-columns with various jump discontinuities was studied in Ref. [18]. The main attention in the paper was paid to the role of jump discontinuities in static mechanical properties. The dynamical properties of a branched Timoshenko beam (which is a generalization of the Euler-Bernoulli beam) were studied in a seminal work [19]. The branched structure of the beam was modeled as a set of zero-range potentials and normal modes and frequencies of the beam were calculated.

The paper is organized as follows:

In Section 2 the model is presented. Time-harmonic vibrations are considered. The mass distribution along the beam is composed of constant term plus a singular term. Also a model example of elastically coupled beams corresponding to a generalized mass distribution are introduced in Appendix A. Four different singularities are considered.

In Section 3 we solve the scattering problem for the four potential cases: $\delta$, $\delta^{\prime}, \delta^{\prime \prime}$, and $\delta^{\prime \prime \prime}$, using both integration procedure and regularization techniques. The corresponding discontinuities of deflection, slope, bending moment, and shear force are surveyed.

Section 4 contains our conclusions.

\section{The model}

Free vibrations, $\psi(x, t)$, on a beam are governed by the Euler-Bernoulli equation [17]. We thus consider equation

$$
\left.-\frac{\partial^{2}}{\partial x^{2}}\left(E I(x) \frac{\partial^{2} \psi(x, t)}{\partial x^{2}}\right)\right)=m(x) \frac{\partial^{2} \psi(x, t)}{\partial t^{2}},
$$

where $E(x), I(x)$, and $m(x)$ denote the elastic modulus of the beam material, the second moment of the area, which must be calculated with respect to the centroidal axis perpendicular to the applied loading, and the mass distribution, respectively, along the beam. The beam is modelled as a one-dimensional object. Without any lateral loading 
the beam occupies the neutral line. The deflection from this line, at position $x$ and time $t$, is given by $\psi(x, t)$. Furthermore the slope of the beam is given by $\partial \psi(x, t) / \partial x$, the bending moment in the beam by $E I \partial^{2} \psi(x, t) / \partial x^{2}$, and shear force in the beam by $\left.\partial\left(E I \partial^{2} \psi(x, t) / \partial x^{2}\right) / \partial x=E I \partial^{3} \psi(x, t) / \partial x^{3}\right)$, when $E I$ is constant, respectively.

Considering time-harmonic vibrations with eigenfrequency $\omega$ we introduce

$$
\psi(x, t)=\psi(x) \exp (i \omega t)+c . c .
$$

where the deflection $\psi(x)$ satisfies the ordinary fourth order differential equation

$$
\psi^{\prime \prime \prime \prime}(x)=\frac{\omega^{2} m(x)}{E I} \psi(x),
$$

the elastic constant and the moment being constant, i.e $E(x) \equiv E$ and $I(x) \equiv I$, along the beam, and prime denoting differentiation with respect to $x$.

Assuming the mass distribution to consist of a constant contribution, $k^{4} E I / \omega^{2}$ where $k$ becomes the propagation constant in the problems we shall consider, and a varying contribution, $E I V(x) / \omega^{2}$ where $V(x)$ is a scattering potential,

$$
m(x)=\frac{E I}{\omega^{2}}\left(k^{4}+V(x)\right),
$$

and inserting (10) into Eq. (9) we obtain

$$
\psi^{\prime \prime \prime \prime}-V(x) \psi=k^{4} \psi
$$

We shall solve the scattering problems in connection with the equation

$$
\psi^{\prime \prime \prime \prime}-\sigma^{4} V_{j}(x) \psi=k^{4} \psi
$$

for $V_{j}(x)$ chosen as the following singular short-range potential

$$
V_{j}(x)=\delta^{(j)}(x)
$$

with $\sigma^{4}$ being a real constant (written in this manner for convenience) and $\delta^{(j)}(x)$ denoting Dirac's $\delta$-function for $j=0$ and its first, second and third derivative with respect to $x$, for $j=1,2$, and 3 , respectively.

Note that by definition the mass function $m(x)$ must be positive for all $x$. However, it is not the case when in Eq. (13) $j=1,2,3$. In Appendix A we consider a system of elastically coupled beams for which $m(x)$ is replaced by a generalized mass function, $M_{ \pm}(x)$, which (in contrast to $m(x)$ ) may assume positive as well as negative values.

For the $\delta$ and $\delta^{\prime}$ potentials the scattering problems are solved by standard integration techniques, while for the $\delta^{\prime \prime}$ and $\delta^{\prime \prime \prime}$ potentials the results cannot be obtained by integration. Instead a regularization techniques by using a simplest one-parameter piece-wise approach is used. It is known for the second order differential equation (see [13]-[16]) that the scattering data depend on the type of regularization. In the present paper we use the simplest one-parameter piecewise constant regularization. Comparative study of different types of regularization for the fourth-order differential equation will be published elsewhere. 


\section{Problems and solutions}

\subsection{Case I: $V_{0}(x)=\delta(x)$}

Integrating equations (12) and (13) with $j=0$ across the point $x=0$ using the standard formula of generalized function theory we get the condition

$$
\left.\psi^{(3)}(x)\right|_{0-} ^{0+}=\sigma^{4} \psi(0)
$$

Multiplying (12) by $x^{n}(n=1,2,3)$, and integrating across the point $x=0$, we obtain that the function $\psi(x)$ and its two first derivatives are continuos at $x=0$ :

$$
\begin{aligned}
& \psi^{\prime \prime}(0+)=\psi^{\prime \prime}(0-)=\psi^{\prime \prime}(0), \\
& \psi^{\prime}(0+)=\psi^{\prime}(0-)=\psi^{\prime}(0), \\
& \psi(0+)=\psi(0-)=\psi(0) .
\end{aligned}
$$

For $x \neq 0(i . \quad e . V(x) \equiv 0)$ we shall write the field as the sum of an incident, a reflected, and a bounded evanescent field for $x<0$ and a transmitted and a bounded evanescent field for $x>0$. Thus we seek for the solution to (12) in the form

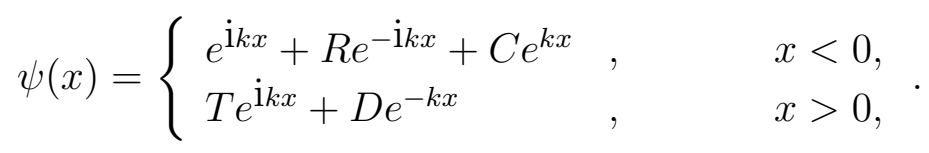

or equivalently,

$$
\psi(x)=\left(e^{\mathrm{i} k x}+R e^{-\mathrm{i} k x}+C e^{k x}\right) H(-x)+\left(T e^{\mathrm{i} k x}+D e^{-k x}\right) H(x) .
$$

where $H(x)$ is the Heaviside step function, $R$ and $T$ are reflection and transmission coefficients, respectively, while $C$ and $D$ denote the amplitudes of the evanescent waves, for $x<0$ and $x>0$, respectively.

It can be shown that the current

$$
j(x) \equiv \psi^{(3)}(x) \psi^{*}(x)-\psi^{(2)}(x) \psi^{(1) *}-\text { c.c. },
$$

with $\psi$ satisfying Eq. (12), is divergence free since

$$
\frac{\partial j(x)}{\partial x}=0
$$

Inserting Eq. (16) into Eq. (19), we find the conservation law

$$
|R|^{2}+|T|^{2}=1
$$

Inserting Eqs. (16) into the conditions (14) and (15) we arrive at the solution

$$
\begin{aligned}
& R=-\frac{(1-\mathrm{i}) \sigma^{4}}{2 \sigma^{4}+4(1+\mathrm{i}) k^{3}}, \quad C=-\frac{(1+\mathrm{i}) \sigma^{4}}{2 \sigma^{4}+4(1+\mathrm{i}) k^{3}} \\
& T=\frac{\left(\sigma^{4}+4 k^{3}\right)(1+\mathrm{i})}{2 \sigma^{4}+4(1+\mathrm{i}) k^{3}}, \text { and } \quad D=C,
\end{aligned}
$$


valid for all values of $k$ and $\sigma$.

The function (17) with the coefficients $R, T, C, D$ given by Eq. (21) is a solution of Eq. (12) with $j=0$ if we understand the product $\delta(x) \psi(x)$ in a common way for the theory of distributions: $\delta(x) \psi(x)=\delta(x) \psi(0)$ :

$$
\psi^{\prime \prime \prime \prime}(x)-\sigma^{4} \delta(x) \psi(0)=k^{4} \psi(x) .
$$

Note that $\psi(x)$ as well as $\psi^{\prime}(x)$ and $\psi^{\prime \prime}(x)$ are continuous ar $x=0$ (see Eqs. (15). We see that $R=\mathcal{O}\left(1 / k^{3}\right)$ and $T=\mathcal{O}(1)$ in the limit $k \rightarrow \infty$.

\subsection{Case II: $V_{1}(x)=\delta^{\prime}(x)$}

Again, by multiplying equation (12) with $x^{n}(n=0,1,2,3)$ and integrating it across the point $x=0$ with the use of standard formula of the generalized function theory, we get the conditions

$$
\begin{gathered}
\left.\psi^{\prime \prime \prime}(x)\right|_{0-} ^{0+}=-\sigma^{4} \psi^{\prime}(0) . \\
\left.\psi^{\prime \prime}(x)\right|_{0-} ^{0+}=\sigma^{4} \psi(0), \\
\psi^{\prime}(0+)=\psi^{\prime}(0-)=\psi^{\prime}(0), \\
\psi(0+)=\psi(0-)=\psi(0) .
\end{gathered}
$$

Inserting Eqs. (16) into the conditions (23) we get the solution

$$
R=\frac{\sigma^{4}\left(i \sigma^{4}+4 k^{2}\right)}{\sigma^{8}+8 k^{4}}, C=-\frac{(1+i) \sigma^{4}\left(\sigma^{4}-2 i k^{2}\right)}{\sigma^{8}+8 k^{4}}, T=\frac{8 k^{4}}{\sigma^{8}+8 k^{4}}, D=\frac{2(1+i) \sigma^{4} k^{2}}{\sigma^{8}+8 k^{4}},
$$

valid for all values of $k$ and $\sigma$.

To verify this result we used also an approach based on the regularization of the

$\delta^{\prime}$-function. We used the simplest piece-wise constant regularization, shown in Figure 1:

$$
V_{1, \varepsilon}(x)=\frac{1}{\varepsilon^{2}}\left\{\begin{array}{rc}
0 & , \quad x<-\varepsilon \\
1 & ,-\varepsilon<x<0 \\
-1 & , 0<x<\varepsilon \\
0 & , \quad x>\varepsilon
\end{array},\right.
$$

such that

$$
V_{1, \varepsilon}(x) \rightarrow \delta^{\prime}(x) \quad \text { as } \quad \varepsilon \rightarrow 0
$$

By writing the solution to Eqs. (12) and (25) in the form

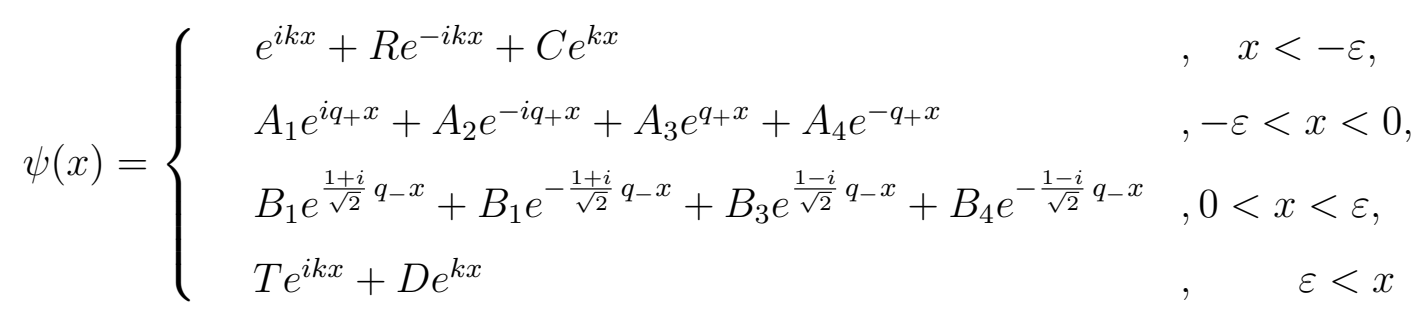




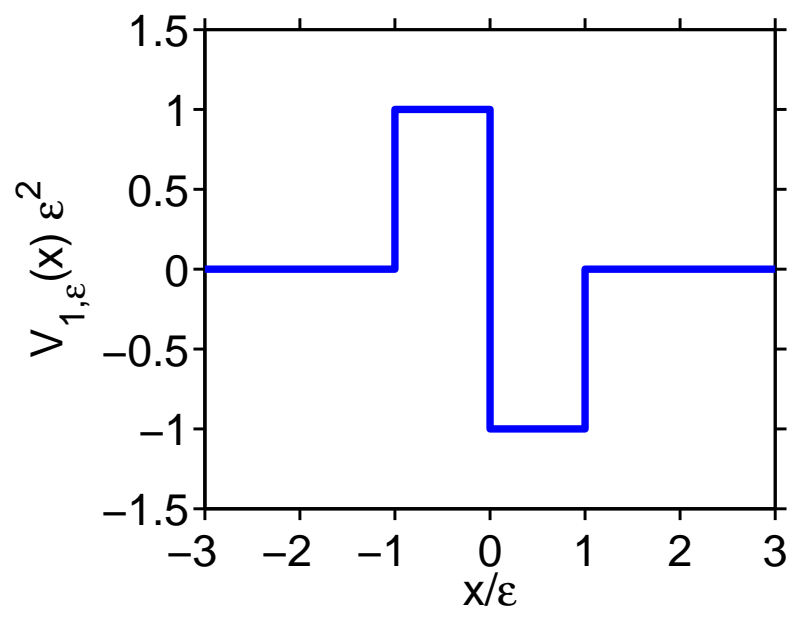

Figure 1. Regularization function, $V_{1, \varepsilon}(x)$

with

$$
q_{ \pm}=\left(\sigma^{4} \varepsilon^{-2} \pm k^{4}\right)^{1 / 4}
$$

Requiring continuity of $\psi^{(n)}(x)(n=0,1,2,3)$ at $x=-\varepsilon, x=0$, and $x=\varepsilon$ we obtain 12 equations for the 12 coefficients, $C, D, R, T, A_{l}, B_{l}(l=1,2,3,4)$. By solving them in the limit $\varepsilon \rightarrow 0$, we obtain that the coefficients $C, D, R, T$ obtained in the framework of the regularization procedure coincide with the corresponding coefficients obtained in the framework of the integration procedure given by Eq. (24).

However, in contrast to the previous case $V_{0}(x)$, the function $(17)$ with the coefficients $R, T, C, D$ given by Eq. (24) is not a solution of Eq. (12) with $V_{1}(x)=\delta^{\prime}(x) \psi(x)$ if we understand the product $\delta^{\prime}(x) \psi(x)$ in a conventional way for the theory of distributions: $\delta^{\prime}(x) \psi(x)=-\delta(x) \psi^{\prime}(0)$. But it is a solution of the equation

$$
\psi^{\prime \prime \prime \prime}(x)-\sigma^{4}\left(-\delta(x) \psi^{\prime}(0)+\delta^{\prime}(x) \psi(0)\right)=k^{4} \psi(x)
$$

Note that $\psi(x)$ and $\psi^{\prime}(x)$ are continuous at $x=0$ (see Eqs. (23c), (23d)). We see also that $R=0\left(1 / k^{2}\right)$ and $T=0(1)$ in the limit $k \rightarrow \infty$.

\subsection{Case III: $V_{2}(x)=\delta^{\prime \prime}(x)$}

By multiplying Eq. (12) and (13) with $j=2$ with $x^{n}(n=0,1,2,3)$ and integrating across $x=0$ we get

$$
\begin{gathered}
\left.\psi^{\prime \prime \prime}(x)\right|_{0-} ^{0+}=\sigma^{4} \psi^{\prime \prime}(0), \\
\left.\psi^{\prime \prime}(x)\right|_{0-} ^{0+}=-2 \sigma^{4} \psi^{\prime}(0), \\
\left.\psi^{\prime}(x)\right|_{0-} ^{0+}=\sigma^{4} \psi(0), \\
\left.\psi(x)\right|_{0-} ^{0+}=0
\end{gathered}
$$




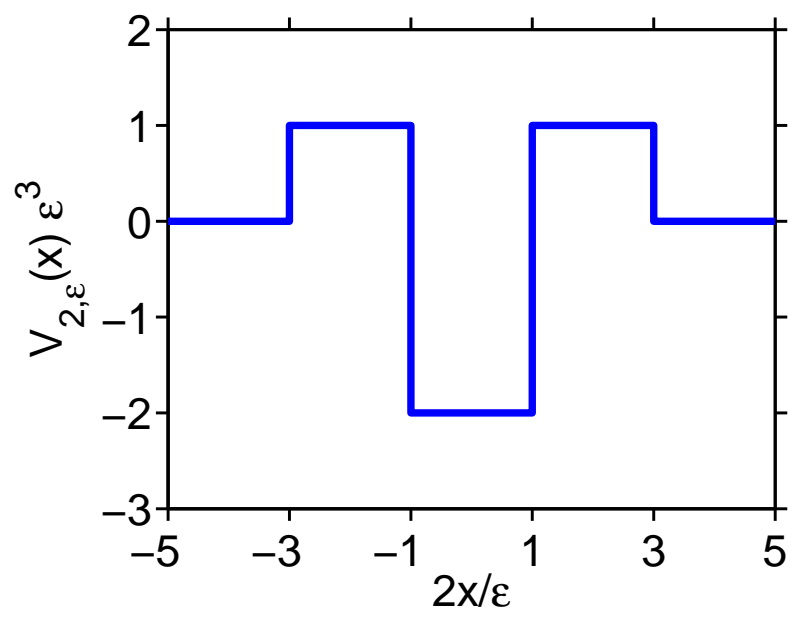

Figure 2. Regularization function, $V_{2, \epsilon}(x)$

To make the set of conditions (30) self-consistent one should in addition to the conditions (30c), (30d) demand that

$$
\begin{aligned}
& \psi(0+)=\psi(0-)=0, \\
& \psi^{\prime}(0+)=\psi^{\prime}(0-)=0
\end{aligned}
$$

It is easily seen that a solution of the form (16) to Eq. (12) with conditions (30a) (30b) and (31) does not exist. Therefore solution by integration is impossible. Instead we regularize the $\delta^{\prime \prime}$ function by replacing $V_{2}(x)$ in Eq. (12) by the barrier-well-barrier system, $V_{2, \varepsilon}(x)$, shown in Figure 2 and given by

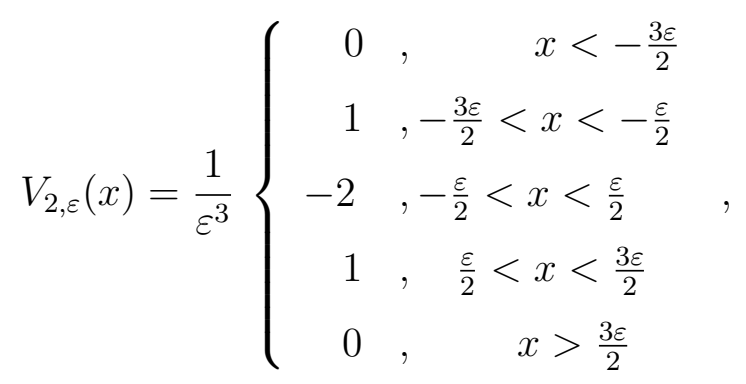

such that

$$
V_{2, \varepsilon}(x) \rightarrow \delta^{\prime \prime}(x) \quad \text { as } \quad \varepsilon \rightarrow 0 .
$$

We shall then write the solution to Eqs. (12) and (32) in the form 


$$
\psi(x)=\left\{\begin{array}{ccc}
e^{i k x}+R e^{-i k x}+C e^{k x} & x<-\frac{3 \varepsilon}{2}, \\
A_{-} \sin q_{1}(x+3 \varepsilon / 2)+B_{-} \cos q_{1}(x+3 \varepsilon / 2) & \\
+C_{-} \sinh \left(q_{1}(x+3 \varepsilon / 2)+D_{-} \cosh \left(q_{1}(x+3 \varepsilon / 2)\right.\right. & ,-\frac{3 \varepsilon}{2}<x<-\frac{\varepsilon}{2}, \\
A_{0} \sin q_{2}(x+\varepsilon / 2)+B_{0} \cos q_{2}(x+\varepsilon / 2) & \\
+C_{0} \sinh \left(q_{2}(x+\varepsilon / 2)+D_{0} \cosh \left(q_{2}(x+\varepsilon / 2)\right.\right. & ,-\frac{-\varepsilon}{2}<x<\frac{\varepsilon}{2}, \\
A_{+} \sin q_{1}(x-\varepsilon / 2)+B_{+} \cos q_{1}(x-\varepsilon / 2) & , \frac{\varepsilon}{2}<x<\frac{3 \varepsilon}{2}, \\
+C_{+} \sinh \left(q_{1}(x-\varepsilon / 2)+D_{+} \cosh \left(q_{1}(x-\varepsilon / 2)\right.\right. & , \quad \frac{3 \varepsilon}{2}<x
\end{array}\right.
$$

with

$$
q_{1}=\left(\frac{\sigma^{4}}{\varepsilon^{3}}+k^{4}\right)^{1 / 4}
$$

and

$$
q_{2}=\left(\frac{1+i}{\sqrt{2}} \frac{2 \sigma^{4}}{\varepsilon^{3}}-k^{4}\right)^{1 / 4} .
$$

Requiring continuity of $\psi^{(n)}(x)(n=0,1,2,3)$ at $x=-3 \varepsilon / 2, x=-\varepsilon / 2, x=\varepsilon / 2$, and $x=3 \varepsilon / 2$ we obtain 16 equations from which the 12 coefficients, $\left.A_{-}, B_{-} \ldots D_{+}\right)$has been eliminated and the remaining four coefficients, R, C, T, and D, can be determined. As a result in the limit $\varepsilon \rightarrow 0$ we get

$$
\begin{aligned}
& R=\frac{-k+\sigma^{4}}{(1+i) k-i \sigma^{4}}, \quad C=\frac{-i k+(i-1) \sigma^{4}}{(1+i) k-i \sigma^{4}}, \\
& T=\frac{i k}{(1+i) k-i \sigma^{4}}, \text { and } D=-T,
\end{aligned}
$$

valid for all values of $k$ and $\sigma$. It is remarkable that in contrast to the $\delta$ - and $\delta^{\prime}$-case when the reflection coefficient $R$ decreases when the wave number $k$ increases, here $R=\mathcal{O}(1)$ and $T=\mathcal{O}(1)$ in the limit $k \rightarrow \infty$. Note that the function (17) with $R, T, C, D$ given by Eq. (37) satisfies the jump conditions (30b), (30c), and (31a) but does not satisfy the condition (31b). Thus $\left.\psi^{\prime \prime}(x)\right|_{0-} ^{0+} \neq 0$, and condition (30a) is therefore meaningless.

\subsection{Case IV: $V_{3}(x)=\delta^{\prime \prime \prime}(x)$}

Integrating equation (12) and (13) with $j=3$ across the point $x=0$ using the standard formula from generalized function theory we immediately get contradictory condition

$$
\left.\psi^{\prime \prime \prime}(x)\right|_{0-} ^{0+}=-\sigma^{4} \psi^{\prime \prime \prime}(0)
$$

Thus Eqs. (12) and (13) cannot be integrated in the usual manner.

Instead we regularize Eq. (12) with $V_{3}(x)=\delta^{\prime \prime \prime}(x)$. We introduce the stretched coordinate $\xi=x / \varepsilon$ and obtain

$$
\frac{d^{4} \psi(\xi)}{d \xi^{4}}-\sigma^{4} \mathcal{V}_{3}(\xi) \psi(\xi)=\varepsilon^{4} k^{4} \psi(\xi),
$$




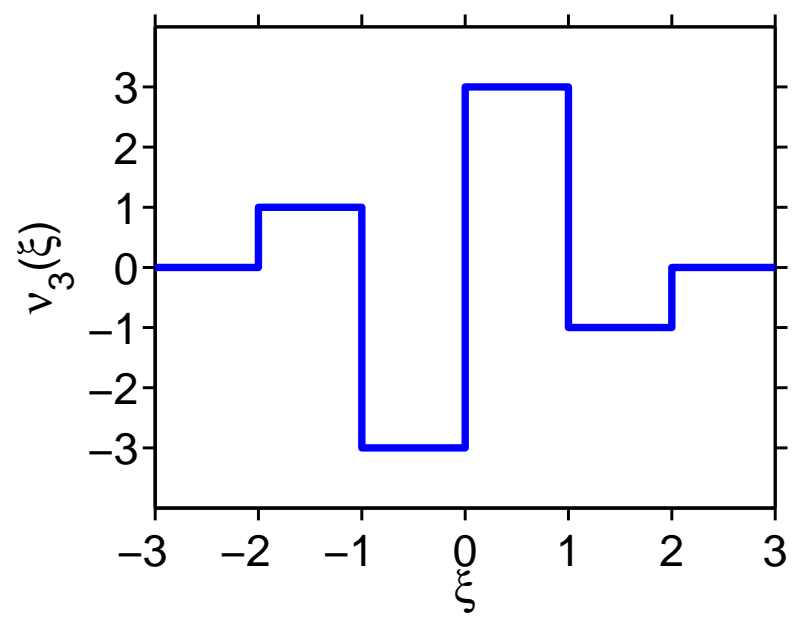

Figure 3. Regularization function, $\mathcal{V}_{3}(\xi)$

where $\mathcal{V}_{3}(\xi)$ now denotes the barrier-well-barrier-well system, $\mathcal{V}_{3}(\xi)$, shown in Figure 3 and given by

$$
\mathcal{V}_{3}(\xi)=\left\{\begin{array}{lc}
0, & \xi<-2, \\
1, & -2<\xi<-1, \\
-3, & -1<\xi<0, \\
3, & 0<\xi<1, \\
-1, & 1<\xi<2, \\
0, & 2<\xi .
\end{array}\right.
$$

We shall then write the solution to Eqs. (12) and (39) with (40) in the form

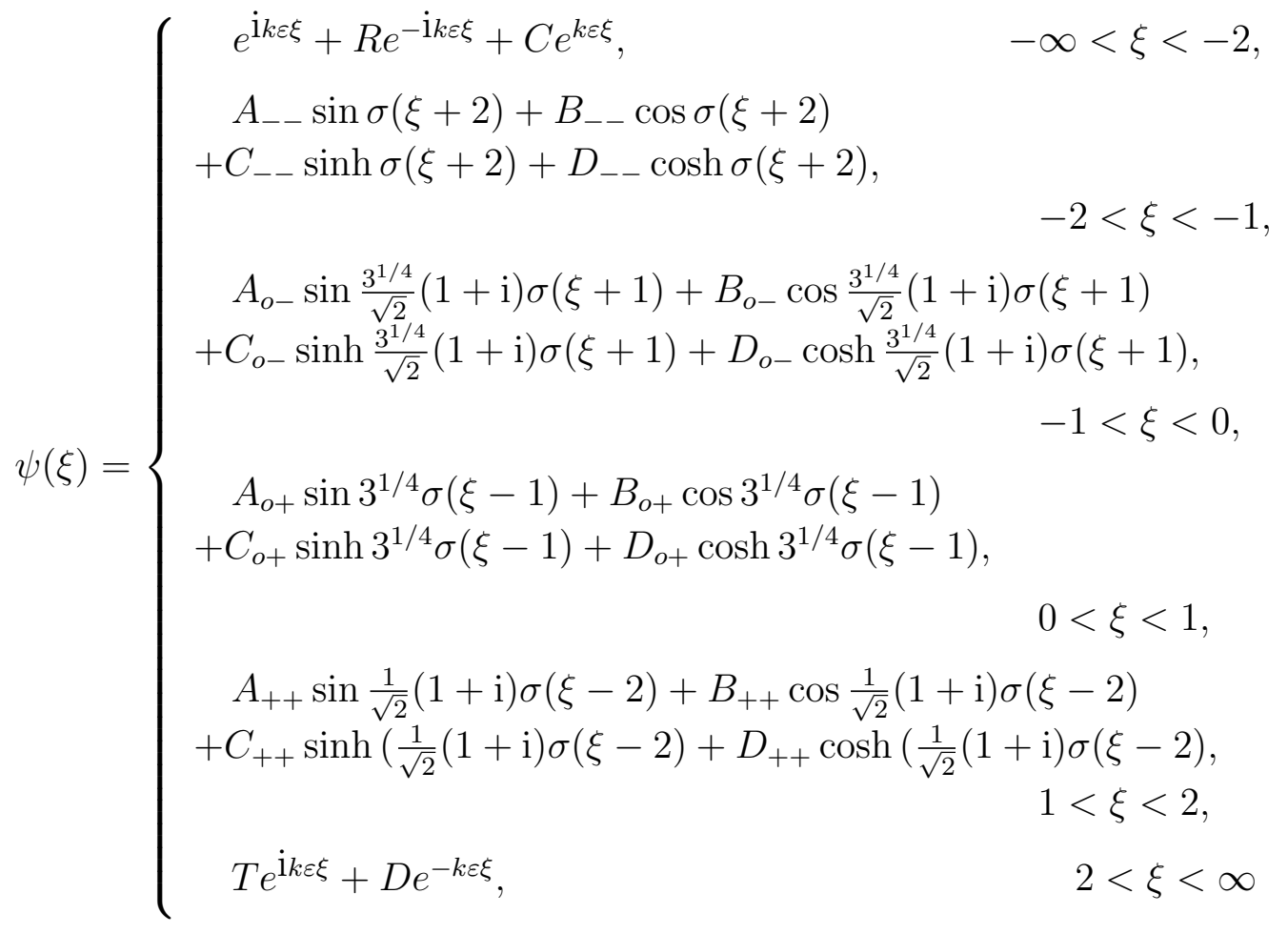

where the 16 arbitrary integration coefficients, $A_{--}, B_{--}, \ldots, D_{++}$must all be expanded 
to four orders in $\varepsilon$

$$
A_{--}=A_{--, 0}+A_{--, 1} \varepsilon+A_{--, 2} \varepsilon^{2}+A_{--, 3} \varepsilon^{3}, \quad \text { e. g. }
$$

Requiring continuity of $\psi^{(n)}(x)(n=0,1,2,3)$ at $x=-2 \varepsilon(\xi=-2)$ and $x=-\varepsilon$ $(\xi=-1)$ and using (41) we obtain 32 conditions from which the 32 constants, $A_{--, 0}, A_{--, 1} \ldots D_{o-, 3}$ can be expressed in terms of $R$ and $C$.

Similarly, requiring continuity $\psi^{(n)}(x)(n=0,1,2,3)$ at $x=\varepsilon(\xi=1)$ and $x=2 \varepsilon$ $(\xi=2)$ we get 32 conditions from which the 32 constants $A_{o+, 0}, A_{o+, 1} \ldots D_{++, 3}$ may be expressed in terms of $T$ and $D$.

Finally, continuity of $\psi^{(n)}(x)(n=0,1,2,3)$ at $x=\xi=0$ leaves us with four equations for $R, C, T$, and $D$ of the form

$$
\mathbf{M} \cdot\left(\begin{array}{c}
R \\
C \\
T \\
D
\end{array}\right)=\mathbf{N}
$$

where $\mathbf{M}$ is the $4 \times 4$ matrix given by

$$
\begin{aligned}
& \mathbf{M}=\left(\begin{array}{cc}
\alpha_{00}-\mathrm{i} \varepsilon \alpha_{01}-\varepsilon^{2} \alpha_{02}+\mathrm{i} \varepsilon^{3} \alpha_{03} & \alpha_{00}+\varepsilon \alpha_{01}+\varepsilon^{2} \alpha_{02}+\varepsilon^{3} \alpha_{03} \\
\alpha_{10}-\mathrm{i} \varepsilon \alpha_{11}-\varepsilon^{2} \alpha_{12}+\mathrm{i} \varepsilon^{3} \alpha_{13} & \alpha_{10}+\varepsilon \alpha_{11}+\varepsilon^{2} \alpha_{12}+\varepsilon^{3} \alpha_{13} \\
\alpha_{20}-\mathrm{i} \varepsilon \alpha_{21}-\varepsilon^{2} \alpha_{22}+\mathrm{i} \varepsilon^{3} \alpha_{23} & \alpha_{20}+\varepsilon \alpha_{21}+\varepsilon^{2} \alpha_{22}+\varepsilon^{3} \alpha_{23} \\
\alpha_{30}-\mathrm{i} \varepsilon \alpha_{31}-\varepsilon^{2} \alpha_{32}+\mathrm{i} \varepsilon^{3} \alpha_{33} & \alpha_{30}+\varepsilon \alpha_{31}+\varepsilon^{2} \alpha_{32}+\varepsilon^{3} \alpha_{33}
\end{array}\right. \\
& \left.-\beta_{00}+\mathrm{i} \varepsilon \beta_{01}+\varepsilon^{2} \beta_{02}-\mathrm{i} \varepsilon^{3} \beta_{03}-\beta_{00}-\varepsilon \beta_{01}-\varepsilon^{2} \beta_{02}-\varepsilon^{3} \beta_{03}\right) \\
& -\beta_{10}+\mathrm{i} \varepsilon \beta_{11}+\varepsilon^{2} \beta_{12}-\mathrm{i} \varepsilon^{3} \beta_{13}-\beta_{10}-\varepsilon \beta_{11}-\varepsilon^{2} \beta_{12}-\varepsilon^{3} \beta_{13} \\
& -\beta_{20}+\mathrm{i} \varepsilon \beta_{21}+\varepsilon^{2} \beta_{22}-\mathrm{i} \varepsilon^{3} \beta_{23}-\beta_{20}-\varepsilon \beta_{21}-\varepsilon^{2} \beta_{22}-\varepsilon^{3} \beta_{23} \\
& \left.-\beta_{30}+\mathrm{i} \varepsilon \beta_{31}+\varepsilon^{2} \beta_{32}-\mathrm{i} \varepsilon^{3} \beta_{33}-\beta_{30}-\varepsilon \beta_{31}-\varepsilon^{2} \beta_{32}-\varepsilon^{3} \beta_{33}\right)
\end{aligned}
$$

and $\mathbf{N}$ is a column vector given by

$$
\mathbf{N}=\left(\begin{array}{c}
-\alpha_{00}-\mathrm{i} \varepsilon \alpha_{01}+\varepsilon^{2} \alpha_{02}+\mathrm{i} \varepsilon^{3} \alpha_{03} \\
-\alpha_{10}-\mathrm{i} \varepsilon \alpha_{11}+\varepsilon^{2} \alpha_{12}+\mathrm{i} \varepsilon^{3} \alpha_{13} \\
-\alpha_{20}-\mathrm{i} \varepsilon \alpha_{21}+\varepsilon^{2} \alpha_{22}+\mathrm{i} \varepsilon^{3} \alpha_{23} \\
-\alpha_{30}-\mathrm{i} \varepsilon \alpha_{31}+\varepsilon^{2} \alpha_{32}+\mathrm{i} \varepsilon^{3} \alpha_{33}
\end{array}\right) .
$$

Here the 32 coefficients, $\alpha_{m n}$ and $\beta_{m n}, m=0,1,2,3, n=0,1,2,3$, are proportional to $k^{n}$. The parameters $\alpha$ and $\beta$ depend on lengthy expressions in terms of elementary functions, i. e. powers of $\sigma$, sin, cos, sinh, and $\cosh$ of $\sigma$ and $3^{1 / 4} \sigma / \sqrt{2}$, and of $3^{1 / 4} \sigma$ and $\sigma / \sqrt{2}$, respectively. These expresions, for which we shall present numerical calculations below, have been obtained by Mathematica.

The determinant of the coefficient matrix $\mathbf{M}$ becomes

$$
|\mathbf{M}| \equiv M=\varepsilon^{2} M_{2}+\varepsilon^{3} M_{3}
$$

where $M_{2}$ depends on $\sigma$ in a complicated manner and therefore is not presented here. 


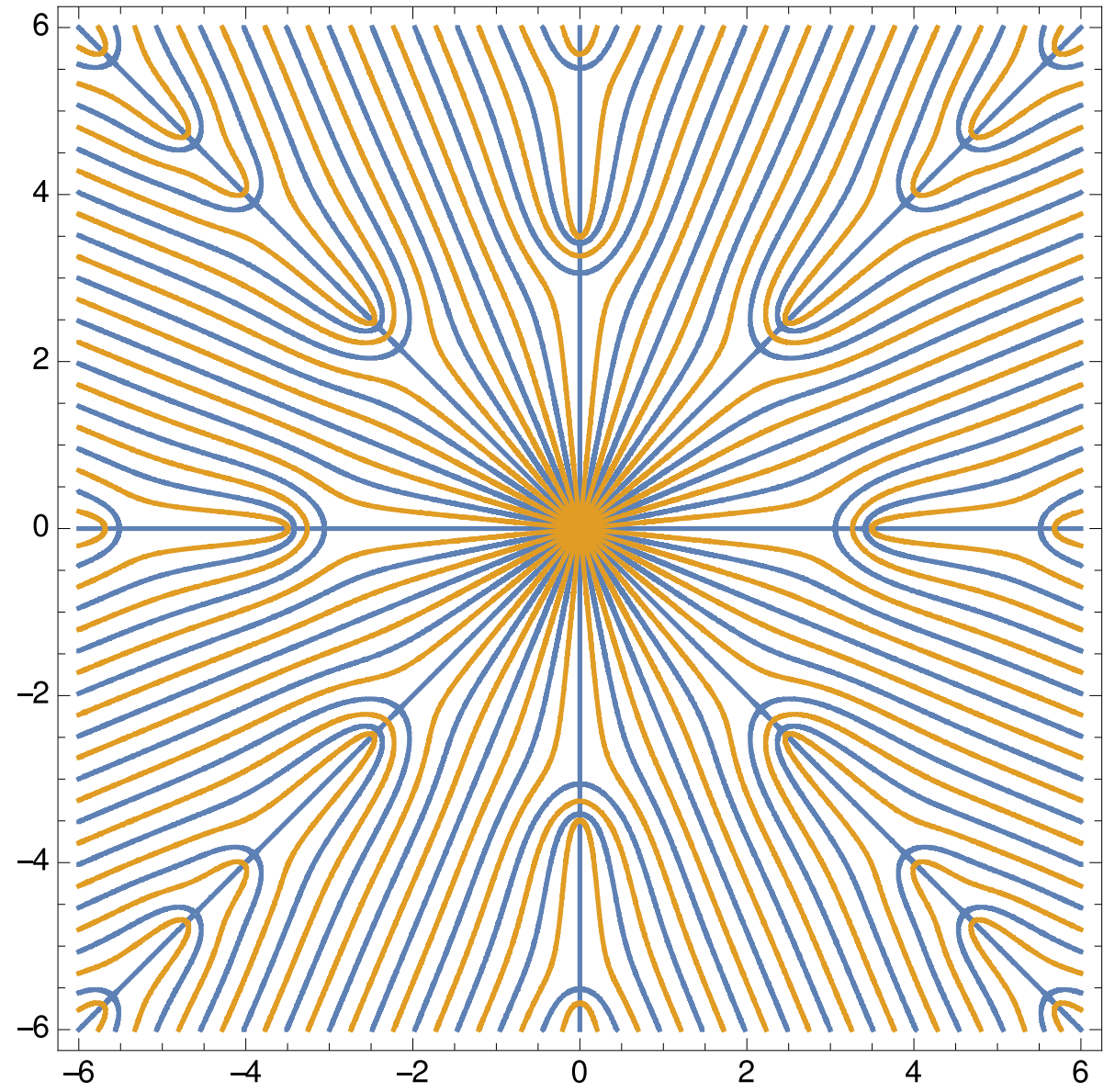

Figure 4. Contour lines $\operatorname{Re}\left[M_{2}(\sigma]=0\right.$ (blue) and $\operatorname{Im}\left[M_{2}(\sigma]=0\right.$ (yellow) in the complex $\sigma$ - plane

Figure 4 is a plot of the contour lines $\operatorname{Re}\left[M_{2}(\sigma)\right]=0$ (blue) and $\operatorname{Im}\left[M_{2}(\sigma)\right]=0$ (yellow) in the complex $\sigma$ - plane, obtained by Mathematica. The zeros of $M_{2}$, determined as the intersection points between the blue and yellow curves are seen to occur symmetrically on the real axis, the imaginary axis, and the bisection lines at the same distance from origo such that the four intersection points on the real and imaginary axes give the same value of $\sigma^{4}=\sigma_{\text {res }}^{4}$, while $\sigma^{4}=\sigma_{\text {res }}^{4}$ for the four intersection points on the bisection lines give the same value with negative sign. Thus resonances are found for both positive and negative values of $\sigma^{4}$. Numerical calculations using $M_{3}$, which contains 48 terms, will be presented below. Solving the linear equations (43) by Cramer's rule we obtain

$$
R=N_{R} / M, \quad C=N_{C} / M, \quad T=N_{T} / M, \quad \text { and } D=N_{D} / M,
$$

where the numerator determinants become

$$
\begin{aligned}
& N_{R}=\mathrm{i} \varepsilon^{2} M_{2}+\varepsilon^{3} N_{R, 3}, \quad N_{C}=-(1+\mathrm{i}) \varepsilon^{2} M_{2}+\varepsilon^{3} N_{C, 3}, \\
& N_{T}=\varepsilon^{3} N_{T, 3}, \quad \text { and } N_{D}=\varepsilon^{3} N_{D, 3} .
\end{aligned}
$$

Like $M$, both $N_{R}$ and $N_{C}$ are zero to zero'th and first order in $\varepsilon$, while $N_{T}$ and $N_{D}$ 
Scattering of flexural waves

\begin{tabular}{cccccc} 
Number $n$ & $\sigma_{\text {res }, n}$ & $\sigma_{r e s, n}^{4}$ & $R_{r, n}$ & $R_{i, n}$ & $\left|T_{n}\right|$ \\
\hline 1 & 3.2629 & 113.34 & -0.58624 & 0.41376 & 0.69651 \\
2 & 3.4951 & 149.22 & -0.99936 & 0.00064 & 0.03577 \\
3 & 5.6830 & 1043.06 & -0.42977 & 0.57023 & 0.70010 \\
4 & 6.6590 & 1966.28 & -1.00000 & $10^{-9}$ & 0.00005 \\
5 & 8.0701 & 4241.39 & 0,00000 & 0.99999 & 0.00344 \\
6 & 9.7995 & 9221.21 & -1.00000 & $10^{-16}$ & $10^{-8}$
\end{tabular}

Table 1. Resonant reflection at $V_{3}=\delta^{\prime \prime \prime}(x)$

become zero to zero'th, first, and second order in $\varepsilon$. Like $M_{3}, N_{R, 3}, N_{C, 3}, N_{T, 3}$, and $N_{D, 3}$ contain 24 terms and will be used in the following numerical calculations.

As a result

$$
\begin{aligned}
& R=\frac{\mathrm{i} M_{2}+\varepsilon N_{R, 3}}{M_{2}+\varepsilon M_{3}}, \quad C=\frac{-(1+\mathrm{i}) M_{2}+\varepsilon N_{C, 3}}{M_{2}+\varepsilon M_{3}}, \\
& T=\frac{\varepsilon N_{T, 3}}{M_{2}+\varepsilon M_{3}}, \quad \text { and } \quad D=\frac{\varepsilon N_{D, 3}}{M_{2}+\varepsilon M_{3}} .
\end{aligned}
$$

For $\varepsilon \rightarrow 0$ we get away from resonances $\left(\sigma \neq \sigma_{\text {res }}, i\right.$. e. $\left.M_{2} \neq 0\right)$

$$
\begin{aligned}
& R=\mathrm{i}, \quad C=-(1+\mathrm{i}) \\
& T=0 \text {, and } D=0 \text {, }
\end{aligned}
$$

while for $\varepsilon \rightarrow 0$ at resonances $\left(\sigma=\sigma_{\text {res }}, i\right.$. e. $\left.M_{2}=0\right)$

$$
\begin{aligned}
& R=\frac{N_{R, 3}}{M_{3}}, \quad C=\frac{N_{C, 3}}{M_{3}} \\
& T=\frac{N_{T, 3}}{M_{3}}, \text { and } \quad D=\frac{N_{D, 3}}{M_{3}} .
\end{aligned}
$$

Using the Mathematica expressions for $M_{2}$, we have calculated the first six zeroes, $\sigma_{n}(n=1,2 \ldots 6)$ on the real axis of the $\sigma$ plane. In Table 1 these values and the corresponding values of $\sigma_{n}^{4}$ are listed. The real and imaginary parts of the corresponding reflection coefficient and the absolute value of the corresponding transmission coefficient, calculated from Eq. (48), are also included. The numerical calulations using Eqs. (48) yield for $\sigma_{n}$ lying on the real or the imaginary axis (i. e. $\sigma_{n}^{4}>0$ )

$$
\begin{array}{ll}
R_{i, n}=1+R_{r, n} & C_{r, n}=C_{i, n}=-1-R_{r, n} \\
T_{r, n}=T_{i, n}=\mp \sqrt{-R_{r, n}\left(1+R_{r, n}\right)} & D_{r, n}=D_{i, n}= \pm \sqrt{-R_{r, n}\left(1+R_{r, n}\right)},
\end{array}
$$

where upper (lower) sign turns out to apply for $n=1,3,5$, and 6 ( $n=2$ and 4). Subscript $r(i)$ denotes the real (imaginary) part.

Similarly, for $\sigma_{n}$ lying on the bisection lines (i. e. $\sigma_{n}^{4}<0$ ) we obtain the real and imaginary parts of the coefficients, now denoted by bar

$$
\begin{array}{ll}
\bar{R}_{r, n}=-1-R_{r, n} \quad \bar{R}_{i, n}=-R_{r, n} & \bar{C}_{r, n}=\bar{C}_{i, n}=R_{r, n} \\
\bar{T}_{r, n}=\bar{T}_{i, n}=\mp \sqrt{-R_{r, n}\left(1+R_{r, n}\right)} & \bar{D}_{r, n}=\bar{D}_{i, n}= \pm \sqrt{-R_{r, n}\left(1+R_{r, n}\right)} .
\end{array}
$$


We note that the conservation equation for the energy current (20) is satisfied in all cases. For all values of $k$ and $\sigma$ away from the resonances, $R=0(1)$ and $T=0$. It is seen in Table 1, that the $\delta^{\prime \prime \prime}$ potential - after the first few resonances - is decreasingly penetrable for higher resonance numbers. Thus, at $n=6\left|T_{n}\right|$ is down to $10^{-8}$, while $R$ rapidly approaches -1 or $i$, corresponding to a perfectly reflecting potential.

We note that it was shown in [9] for the $\delta^{\prime}$ potential in the Schrödinger equation (1) the reflection coefficient $R$, rapidly approaches -1, also corresponding to a perfectly reflecting potential, for higher resonances.

\subsection{Summary}

In Table 2 we list the methods used in the previous sections as well as the resulting jumps in $\psi(x), \psi^{\prime}(x), \psi^{\prime \prime}(x)$, and $\psi^{\prime \prime \prime}(x)$ which we obtain using (16) for $x \rightarrow 0-$ and $0+$, i. e.

$$
\begin{aligned}
& \left.\psi(x)\right|_{0-} ^{0+}=T+D-1-R-C, \\
& \left.\psi^{\prime}(x)\right|_{0-} ^{0+}=i k(T+i D-1+R+i C), \\
& \left.\psi^{\prime \prime}(x)\right|_{0-} ^{0+}=(i k)^{2}(T-D-1-R+C), \\
& \left.\psi^{\prime \prime \prime}(x)\right|_{0-} ^{0+}=(i k)^{3}(T-i D-1+R-i C),
\end{aligned}
$$

inserting the scattering coefficients from (21), (24), (37), (47) and (49).

We see that the $\delta$ scattering potential (Case 1) corresponds to a discontinuity of the shear force at $x=0$, while deflection, slope, and bending moment are continuous. For the $\delta^{\prime}$ scattering potential (Case 2) bending moment as well as shear force become discontinuous while deflection and slope are continuous. Similar conditions are obtained for the $\delta^{\prime \prime}$ scattering potential (Case 3) and the $\delta^{\prime \prime \prime}$ scattering potential (Case 4 ) in the non-resonant case, while only the deflection is continuous when the latter potential is resonant.

\section{Conclusions}

Beam vibrations are governed by the fourth order Euler-Bernoulli equation. We study the scattering of such time-harmonic vibrations by short-range localized imperfections in the mass density distribution of on an otherwise homogeneous beam. Also the scattering by a singularity short-range defect in the position dependant coupling between two beams is considered. The singularity defects are modeled by a Dirac delta function and its derivative up to order three. The physical interpretation of these scattering problems is outlined in the summary at the end of the preceding section.

In the $\delta(x)$ case, the scattering problem is solved by integration of the differential equation across the singularity point. For an incident wave the reflection and transmission coefficients, $R$ and $T$, for the reflected and transmitted waves are determined. Evanescent waves, damped in the directions of incidence and of transmission, are also generated at the scatterer, and their amplitude coefficients, $C$ and 


\begin{tabular}{ccccc} 
Section & Method & $V(x)$ & $\left.\psi(x)\right|_{0-} ^{0+}$ & $\left.\psi^{\prime}(x)\right|_{0-} ^{0+}$ \\
\hline 3.1 & int. & $\sigma^{4} \delta(x)$ & 0 & 0 \\
3.2 & int. & $\sigma^{4} \delta^{\prime}(x)$ & 0 & 0 \\
& and reg. & & & 0 \\
3.3 & reg. & $\sigma^{4} \delta^{\prime \prime}(x)$ & 0 & 0 \\
3.4 & reg.: & $\sigma^{4} \delta^{\prime \prime \prime}(x)$ & & $2 \mathrm{i} k\left(R_{r, n} \mp \sqrt{-R_{r, n}\left(1+R_{r, n}\right)}\right)$
\end{tabular}

\begin{tabular}{ccccc} 
Section & Method & $V(x)$ & $\left.\psi^{\prime \prime}(x)\right|_{0-} ^{0+}$ & $\left.\psi^{\prime \prime \prime}(x)\right|_{0-} ^{0+}$ \\
\hline 3.1 & int. & $\sigma^{4} \delta(x)$ & 0 & $\frac{2(1+\mathrm{i}) k^{3} \sigma^{4}}{2(1+\mathrm{i}) k^{3}+\sigma^{4}}$ \\
3.2 & int. & $\sigma^{4} \delta^{\prime}(x)$ & $\frac{8 k^{4} \sigma^{4}+2(1+\mathrm{i}) k^{2} \sigma^{8}}{8 k^{4}+\sigma^{8}}$ & $\frac{-8 \mathrm{i} k^{5} \sigma^{4}+2(1+\mathrm{i}) k^{3} \sigma^{8}}{8 k^{4}+\sigma^{8}}$ \\
& and reg. & $\frac{2(1-\mathrm{i}) k^{2} \sigma^{4}}{(1+\mathrm{i}) k-\mathrm{i} \sigma^{4}}$ & $2(1+\mathrm{i}) k^{3}$ \\
3.3 & reg. & $\sigma^{4} \delta^{\prime \prime}(x)$ & $2(1+\mathrm{i}) k^{2}$ & $2(1+\mathrm{i}) k^{3}$ \\
3.4 & reg.: & $\sigma^{4} \delta^{\prime \prime \prime}(x)$ & $2(1+\mathrm{i}) k^{2}\left(1+R_{r, n}\right.$ & $2 k^{3}\left((1+\mathrm{i})+R_{r, n}\right.$ \\
& non-res. & & $\left.\pm \sqrt{-R_{r, n}\left(1+R_{r, n}\right)}\right)$ & $\left.\mp \sqrt{-R_{r, n}\left(1+R_{r, n}\right)}\right)$
\end{tabular}

Table 2. Resulting jump conditions at $x=0$.

(Abbreviations: int. = integration, reg. = regularization, res. = resonant).

$R_{r, n}$ is the real part of the reflection coefficient at the $n$ 'th resonance. Numerical values of $R_{r, n}$ for $n=1,2, \ldots 6$ are listed in Table 1 .

$D$, are determined. In contrast to scattering problems at the second order differential equation we here observe coexisting travelling and localized waves. It is also shown that the corresponding wave function satisfies a differential equation where the $\delta(x)$-potential can be considered as a conventional generalized function.

In the $\delta^{\prime}(x)$ case the scattering problem is solved both by integration and by using regularization procedure. The results coincide. It is also shown that the corresponding wave function satisfies a differential equation with the $\delta^{\prime}(x)$-potential which is understood as $\delta^{\prime}(x) \psi(x)=\delta^{\prime}(x) \psi(0)-\delta(x) \psi^{\prime}(0)$.

In the $\delta^{\prime \prime}(x)$ and $\delta^{\prime \prime \prime}(x)$ cases, the integration approach cannot be used. The problems are then regularized by replacing the $\delta^{\prime \prime}(x)$ and $\delta^{\prime \prime \prime}(x)$ by sequences of rectangular approximations using a one-parameter piece-wise constant approach.

Different dependencies, of $R$ and $T$, in the limit $k \rightarrow \infty$ are noted in the $\delta(x), \delta^{\prime}(x)$ and $\delta^{\prime \prime}(x)$ cases.

In the final case, $\delta^{\prime \prime \prime}(x)$, we introduce a stretched variable in the four intervals in which the $\delta^{\prime \prime \prime}(x)$ function is approximated. Expanding the solution in these intervals 
into four orders in $\varepsilon$, we obtain 32 equations for 32 expansion coefficients which are solved with respect to $R, T, C$, and $D$. This immensely complicated task can in praxis only be carried out by a symbolic computer program.

We have used Mathematica for this purpose and found out that in the limit $\varepsilon \rightarrow 0$ the $\delta^{\prime \prime \prime}$-barrier is opaque (the transmission coefficient $T$ vanishes) for all values of the potential strength $\sigma$ except a countable set of values $\sigma_{n}(n=1,2, \ldots)$ (resonances) for which the barrier is partially transparent and the transmission coefficient $T$ is finite. The resonance values of the potential strength $\sigma$ and the corresponding scattering data, $R, T, C$, and $D$, are then determined numerically.

Outside the resonance the scatterer is thus totally reflecting with an evanescent wave in front, for all values of the wave number $k$. For the smallest $\sigma_{n}$ the scatterer turns out to be partially penetrable. However, already after a few resonances the scatterer for $\sigma=\sigma_{n}$ becomes almost perfectly reflecting.

\section{Acknowledgements}

A. V. Zolotaryuk and J. Juul Rasmussen are thanked for helpful suggestions and discussions. Yu. B. G. thanks Department of Mathematics and Department of Physics, Technical University of Denmark for hospitality and acknowledges financial support from Civilingeniør Frederik Leth Christiansens Almennyttige Fond. Yu. B. G. also acknowledges partial financial support from special grant "Structure and dynamics of statistical and quantum-field systems" (0117U00240) NAS of Ukraine.

\section{Appendix A. Coupled Beams. A model example}

The Lagrange function for the beam (B) corresponding to Eq. (7) can be written

$$
L_{B}=\frac{1}{2} \int d x\left[m(x)\left(\frac{\partial \psi}{\partial t}\right)^{2}-E I(x)\left(\frac{\partial^{2} \psi}{\partial x^{2}}\right)^{2}\right] .
$$

We also consider two identical elastically coupled beams (CB) with vibrations, $\psi_{1}(x, t)$ and $\psi_{2}(x, t)$. The Lagrangian for this system can be written

$$
L_{C B}=\frac{1}{2} \int d x\left[m(x)\left(\left(\frac{\partial \psi_{1}}{\partial t}\right)^{2}+\left(\frac{\partial \psi_{2}}{\partial t}\right)^{2}\right)-E I(x)\left(\left(\frac{\partial^{2} \psi_{1}}{\partial x^{2}}\right)^{2}+\left(\frac{\partial^{2} \psi_{2}}{\partial x^{2}}\right)^{2}\right)-2 \alpha(x) \psi_{1} \psi_{2}\right],
$$

where $\alpha(x)$ is a position dependant coupling between the two beams. Introducing inphase and out-of-phase modes

$$
\psi_{ \pm}=\frac{1}{\sqrt{2}}\left(\psi_{1} \pm \psi_{2}\right)
$$

the Lagrangian $L_{C B}$ may now be written

$$
L_{C B}=\frac{1}{2} \int d x\left[m(x)\left(\left(\frac{\partial \psi_{+}}{\partial t}\right)^{2}+\left(\frac{\partial \psi_{-}}{\partial t}\right)^{2}\right)-E I(x)\left(\left(\frac{\partial \psi_{+}^{2}}{\partial x^{2}}\right)^{2}+\left(\frac{\partial^{2} \psi_{-}}{\partial x^{2}}\right)^{2}\right)-\alpha(x)\left(\psi_{+}^{2}-\psi_{-}^{2}\right)\right] .
$$


The equations of motion for the modes $\psi_{+}$and $\psi_{-}$, given by (A.3) become

$$
\left.-\frac{\partial^{2}}{\partial x^{2}}\left(E I(x) \frac{\partial^{2} \psi_{ \pm}(x, t)}{\partial x^{2}}\right)\right) \mp \alpha(x) \psi_{ \pm}(x, t)=m(x) \frac{\partial^{2} \psi_{ \pm}(x, t)}{\partial t^{2}}
$$

Again, for constant $E(x)=E$ and $I(x)=I$, and time-harmoninic vibrations, Eq. (8), we now get

$$
\psi(x)_{ \pm}^{\prime \prime \prime \prime}(x)=\frac{\omega^{2} M_{ \pm}(x)}{E I} \psi_{ \pm}(x)
$$

where

$$
M_{ \pm}(x) \equiv m(x) \mp \alpha(x) / \omega^{2} .
$$

Here $M_{ \pm}(x)$, even for $m(x)=m$, where $m$ is a positive constant, may assume positive as well as negative values, (depending on the values of $\alpha(x)$ ). For $m=E I k^{4} / \omega^{2}$, we then obtain Eq.(11) with $V(x)=\mp \frac{\alpha(x)}{E I}$.

\section{Bibliography}

[1] Berezin F A and Faddeev L D 1961 Sov. Math. Dokl. 2 372; Engl. transl.: Math. USSR Dokl. 137 1011

[2] Demkov Y N and Ostrovskii V N 1975 Zero-Range Potentials and Their Applications in Atomic Physics (Leningrad: Leningrad University Press)

[3] Demkov Y N and Ostrovskii V N 1988 Zero-Range Potentials and Their Applications in Atomic Physics (New York: Plenum)

[4] Albeverio S, Gesztesy F, Høegh-Krohn R and Holden H 2005 Soluble Models in Quantum Mechanics (With an Appendix by Pavel Exner) 2nd revised edn (Providence: RI: American Mathematical Society: Chelsea Publishing)

[5] Albeverio S and Kurasov 1999 Singular Perturbations of Differentiable Operators: Solvable Schrödinger-Type Operators (Cambridge: Cambridge University Press)

[6] Perez J F and Coutinho F A B 1991Am. J. Phys. 5952

[7] Brasche J F, Figari R and Teta A 1998 Potential Analysis 8163

[8] Exner P, Neidhardt H and Zagrebnov V A 2001 Commun. Math. Phys. 224593

[9] Christiansen P L, Arnbak H C, Zolotaryuk A V, Ermakov V N and Gaididei Y B 2003 J. Phys. A: Math. Gen. 367589

[10] Coutinho F A B and Amaku M 2009 Eur. J. Phys. 301015

[11] Exner P and Manko S S 2014 Lett Math Phys. 1041079

[12] Seba P 1986 Rep. Math. Phys. 24111

[13] Golovaty Yu D and Manko S S 2009 Ukr. Math. Bull. 6173 (arXiv:0909.1034v [math.SP]) (in Ukrainian).

[14] Zolotaryuk A V, Zolotaryuk Y A 2011 J. Phys. A: Math. Theor. 44375305.

[15] Zolotaryuk A V, Zolotaryuk Y A 2015 J. Phys. A: Math. Theor. 48035302.

[16] Zolotaryuk A V 2017 J. Phys. A: Math. Theor. 50225303.

[17] Gere J M and Timoshenko S P 1997 Mechanics of Materials (Boston: PWS Publishing Company)

[18] Yavari A and Sarkani S 2001 Int. J. Mech. Sci. 431543

[19] Shaker F.J, Method and calculating the normal modes and frequencies of a branched Timoshenko beam, 1968 NASA technical note, NASA TN D-4560 